\title{
Design and Development of Textile Sensor for Kyphosis Detection
}

\author{
Yamuna $\mathrm{I}^{\mathrm{a}, 1}$, Abirami $\mathrm{A}^{\mathrm{b}}$, Esther Florence $\mathrm{S}^{\mathrm{b}}$ and Vimal Samsingh $\mathrm{R}^{\mathrm{b}}$ \\ ${ }^{a}$ ECE Department, SSN College of Engineering, Chennai \\ ${ }^{\mathrm{b}}$ Mechanical Department, SSN College of Engineering, Chennai
}

\begin{abstract}
The proposed system is to design and develop a textile sensor. People suffering from kyphosis disease may have a hunchedback appearance due to forward bend in spinal cord. The textile sensor is based on piezoresistivity, where change in resistance is observed due to mechanical bending of the sensor. Thesensor is designed in such a way that it can detect any bend in the spinal cord. The initial design has been developed using a flex sensor. The proposed sensor is made up of five layers in total with a piezoresistive layer sandwiched in between two conductive layers. The top and bottom of the structure is then covered with a dielectric layer to form a complete wearable textile sensor. The performance of the sensor is then analyzed for two outer covers, felt and polyester. The piezoresistive material used in this sensor is velostat. The performance of the flex sensor and the developed piezoresistive textile sensor have been compared and analysed.
\end{abstract}

Keywords. flex sensor, piezoresistive, textile sensor, velostat.

\section{Introduction}

Recent advancements in wearables have introduced a surge in the design and development of wearable sensors. For any wearable device realization, textile based structures have higher compatibility as they are flexible and weigh less when compared with conventional rigid structures. This category of textiles with digital abilities has been recognized as digital textiles (e-textiles). E-textiles are capable of sensing, processing, communication and generation of data. Because of these advantages textile based sensor design and development has emerged as a rapidly evolving area of research. Textile based sensors $[2,6,7,8,11,16]$ provide an interface between the customer and a digital machine by changing physiological or environmental warning signs into electrical alerts. Wearable sensors are usually designed to be operated as pressure sensors [1], stress sensors [10], tactile sensors [6], force sensors [15] etc. Most of the inflexible sensors are utilized in industrial packages. There are very few bendy piezoresistive sensors mentioned in literature. Piezoresistive sensors $[5,6,7,11,12,16]$ have been used for gait analysis [1], angle measurement [2, 5], respiratory tracking [8] etc. The proposed sensor is primarily based on piezoresistivity. Piezoresistive substances show a resistance change because of mechanical strain or bending. Several peizoresistive substances have been studied. The concept is to develop a completely integrated textile sensor exhibiting piezoresistivity to detect, examine and alert the user of Kyphosis. Kyphosis happens in people due to a forward bend in spinal cord with a hunched back appearance as shown

\footnotetext{
${ }^{\text {a1 }}$ Yamuna, Dept of ECE,SSN college of engineering, Chennai

Email: yamunamalliga@gmail.com
} 
in Figure 1. The commercially existing technique to detect of Kyphosis includes Computed Tomography (CT) test and Magnetic Resonance (MR) imaging where in the spinal wire bend is calculated. Also the treatment entails physiotherapy and a special jacket designed to hold the backbone straight. Hence an easily accessible solution for early detection and posture correction at home has been designed and developed.

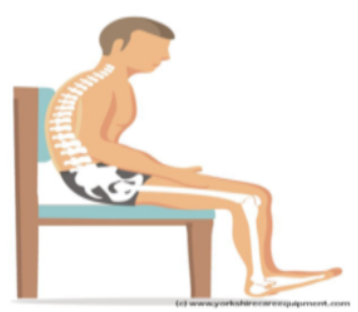

Figure 1. Person with Kyphosis disease (https://www.mayoclinic.org)

\section{Design and Fabrication}

Five layers are incorporated within the fabric sensor. The first and last layer consists of a dielectric outer covering used for mechanical stability of the shape and to prevent any contact with human tissues. The second and fourth layer forms the conductive layer. The conductive layer is introduced to act as terminals for communicating the change in resistance detected in the sensor. The third layer is piezoresistive and is responsible for the actual resistance change of the sensor. Piezoresistive material produces a resistance change while the shape is bent. The piezoresistive material used here is Velostat and it is sandwiched between the two silver conductive textile layers. Fabrication is done by sewing all the layers at the ends to form a complete wearable sensor. The conductive portion alone has two contacts exterior to the outer covering for measuring the resistance change. Fig. 2 shows the layer diagram of textile sensor.

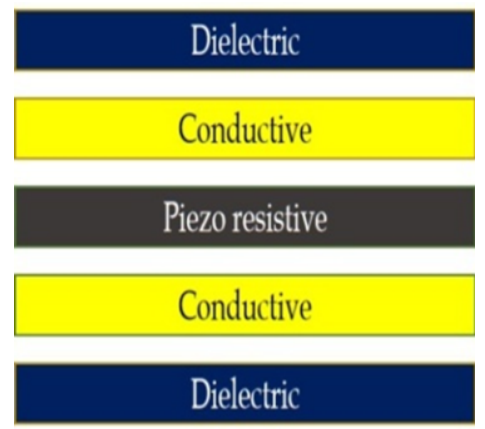

Figure 2. Layers Diagram of Textile sensor.

\section{Proposed System}

To enhance the comfort of the user and also to detect and analyze Kyphosis with ease at home, a novel solution has been proposed. A novel five layered all textile 
piezoresistive sensor has been proposed to detect bend in spinal cord. Piezoresistive materials show a resistance change due to mechanical stress or bending. This property has been exploited to provide a solution for detection of kyphosis. In addition, to compare the performance of the realized sensor, a conventional bend sensor has been used.

\subsection{Analysis using flex sensor}

Flex sensor is a commercially available bend sensor. The flex sensor is bending and the change in resistance has been recorded. The angle and resistances readings obtained have been plotted. Figure 3 (a) shows the flex sensor and figure 3 (b) shows the for resistance angle characteristics for the flex sensor. The graph shows that the there is a resistance change due to mechanical bending of the sensor.

(a)

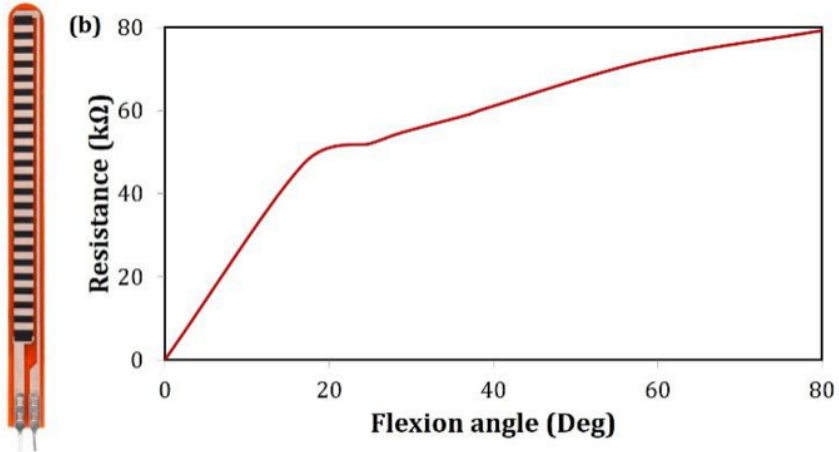

Figure 3. (a) Flex sensor, (b) Resistance-Angle Characteristics using flex sensor.

\subsection{Analysis using Velostat sensor}

Two variants of the proposed sensor has been fabricated and analyzed. The only change made in the proposed sensor is the material used for outer covering. Considering the comfort of the user, polyester and felt were used and compared.

The grey coloured sensor shown in figure 4(a) is the polyester variant while the blue coloured one in figure 4 (b) is the felt variant. On bending, both the sensors showed a change in the resistance. The resistance angle characteristics in figure 4 (c) shows that the sensor realized with felt has better characteristics i.e. it is more linear when compared with the polyester variant. Also the sensor is structurally stable and strong when felt is used. 
(a)

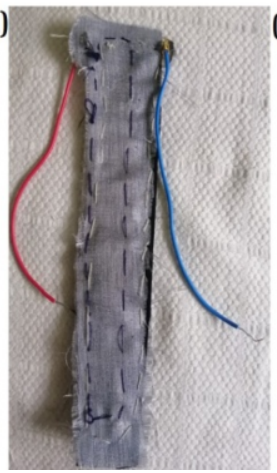

(b)

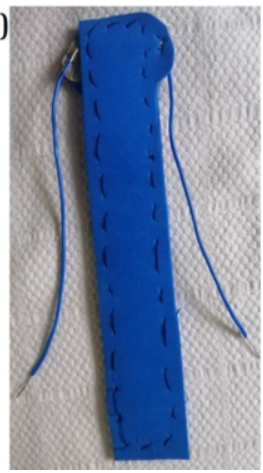

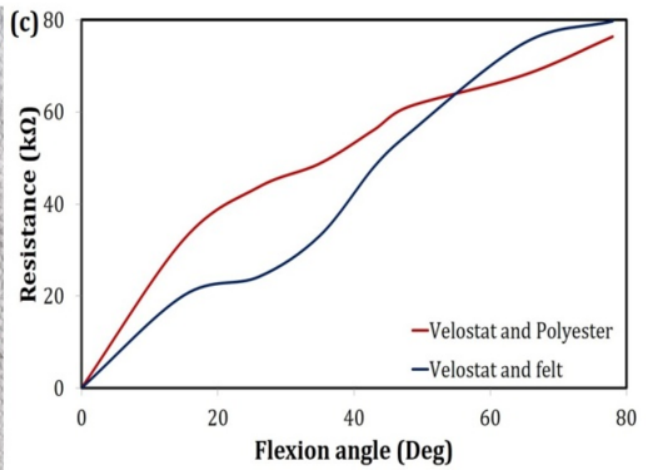

Figure 4. Velostat sensor using (a) polyester and (b) felt(c) Resistance-Angle Characteristics using velostat sensor.

The sensor was subject to bending and twisting for assessing its performance in mechanically deformed conditions, as any wearable is prone to such mechanical deformations.

\subsection{Bend Analysis}

To analyze the performance of the sensor in bent conditions, the sensor was completely folded and left to be automatically be unfolded as shown in figure 5 (a). During this release, the resistance values were recorded. It was noted that, the flex sensor can be bent only till 90 degrees while the proposed sensor was capable of bending up to 180 degrees due to the highly flexible nature of the sensor. It can be seen from the figure $5(\mathrm{~b})$ that the realized sensor has better resistance characteristics than the flex sensor due to better flexibility.
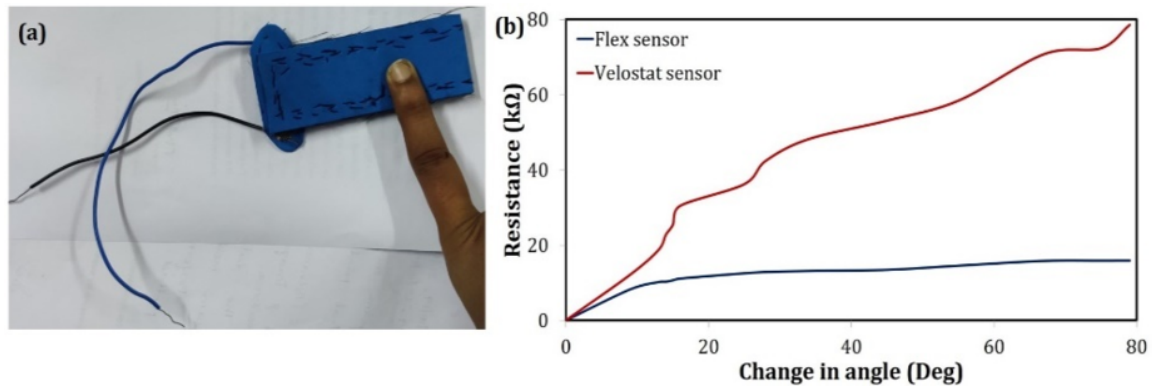

Figure 5. (a) Bending of velostat sensor (b) Resistance-Angle Characteristics using flex and velostat sensor.

\subsection{Twist Analysis}

Another analysis that can be performed on any textile sensor is the twist analysis. Similar to bending analysis, the sensor was twisted completely and then left to be automatically released. The twisted sensor can be seen from figure 6 (a). The 
resistance values recorded can be seen from figure 6 (b). The flex sensor cannot be twisted like the realized piezoresistive textile sensor as it is rigid in nature. The resistance angle characteristics prove that the performance was better for the textile sensor.

Figure 6. (a) Twisting of velostat sensor (b) Resistance-Angle Characteristics using flex and velostat sensor.

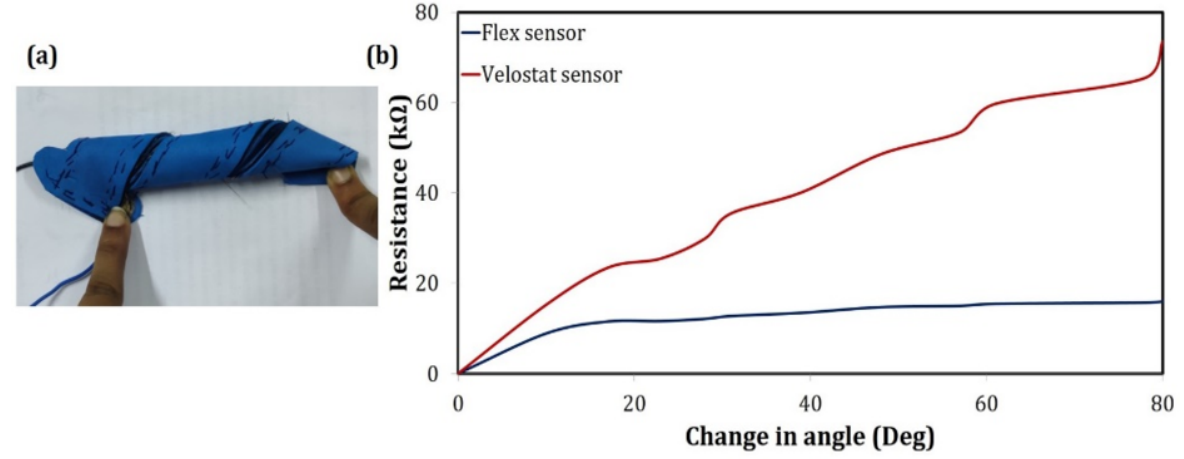

Bending and twisting analysis prove that irrespective of the deformations, the sensor works perfectly. This proves that the sensor is a perfect candidate for being used as a wearable.

\section{Conclusion}

A completely integrated all textile novel piezoresistive sensor has been realized. A comparison with a commercially available sensor as done to assess the functionality of the proposed sensor. The overall performance of the sensor has been validated using practical evaluation of the sensor. The proposed sensor is lightweight, cost effective and with a low profile. The main application of the sensor is to analyze any bend in the spinal cord for early detection of kyphosis. The change in resistance has been used to achieve the necessary detection. In addition deformation analysis proves that the sensor is perfect for being used as a wearable.

\section{Acknowledgment}

This work was supported in part by the DST SERB under Grant No. SB/S3/EECE/055/2016.

\section{References}

[1] G. Li, T. Liu and J. Yi (2018), Wearable sensor system for detecting gait parameter of abnormal gaits: a feasibility study . in IEEE Sensors Journal, vol. 18, no. 10, pp. 4234-424.

[2] A. Grassi, F. Cecchi, M. Maselli, M. Röling (2017), C. Laschi and M.Cianchetti, Warp-knitted textile as a strain sensor: characterization procedure and application in a comfortable wearable goniometer, in IEEE Sensors Journal, vol. 17, no. 18, pp. 5927-5936.

[3] S. C. Mukhopadhyay (2015), Wearable sensors for human activity monitoring: a review. in IEEE Sensors Journal, vol. 15, no. 3, pp. 1321-1330.

[4] M. M. Rodgers, V. M. Pai and R. S. Conroy (2015), Recent advances in wearable sensors for health monitoring. in IEEE Sensors Journal, vol. 15, no. 6, pp. 3119-3126. 
[5] F. Lorussi, S. Galatolo and D. E. De Rossi (2009), Textile-based electrogoniometers for wearable posture and gesture capture systems . in IEEE Sensors Journal, vol. 9, no. 9, pp. 1014-1024.

[6] S. K. Kirthika, G. Ponraj and H. Ren (2017), Fabrication and comparative study on sensing characteristics of soft textile-layered tactile sensors. in IEEE Sensors Letters, vol. 1, no. 3, pp. 1-4.

[7] X. Lin and B. Seet (2015), A linear wide-range textile pressure sensor integrally embedded in regular fabric.in IEEE Sensors Journal, vol. 15, no. 10, pp. 5384-5385.

[8] S. D. Min, Y. Yun and H. Shin (2014), Simplified structural textile respiration sensor based on capacitive pressure sensing method in IEEE Sensors Journal, vol. 14, no. 9, pp. 3245-3251.

[9] J. Witt et al (2012), Medical textiles with embedded fiber optic sensors for monitoring of respiratory movement . in IEEE Sensors Journal, vol. 12, no. 1, pp. 246-254.

[10] T. Hoffmann, B. Eilebrecht and S. Leonhardt (2011), Respiratory monitoring system on the basis of capacitive textile force sensors. in IEEE Sensors Journal, vol. 11, no. 5, pp. 1112-1119.

[11] C. Massaroni et al (2019). Smart textile based on piezoresistive sensing elements for respiratory monitoring. in IEEE Sensors Journal, vol. 19, no. 17, pp. 7718-7725, 1 Sept.1, 2019.

[12] A. Oliveri, M. Maselli, M. Lodi, M. Storace and M. Cianchetti (2019), Model-based compensation of rate-dependent hysteresis in a piezoresistive strain sensor. in IEEE Trans. Industrial Electronics, vol. 66 , no. 10 , pp. 8205-8213.

[13] M. O. F. Emon, J. Lee, U. H. Choi (2019), D. Kim, K. Lee and J. Choi, Characterization of a soft pressure sensor on the basis of ionic liquid concentration and thickness of the piezoresistive layer. in IEEE Sensors Journal, vol. 19, no. 15, pp. 6076-6084.

[14] D. Franke, S. Binder and G. Gerlach (2017), Performance of fast-responsive, porous crosslinked poly(n-isopropylacrylamide) in a piezoresistive microsensor .in IEEE Sensors Letters, vol. 1, no. 6, pp. $1-4$.

[15] B. W. Lee and H. Shin (2016), Feasibility study of sitting posture monitoring based on piezoresistive conductive film-based flexible force sensor in IEEE Sensors Journal, vol. 16, no. 1, pp. 15-16.

[16] J. Deignan et al (2016), Textile piezoresistive sensors for on-body measurement of spinal extension . IEEE SENSORS, Orlando, pp. 1-3.

[17] A. R. Anusha, A. L. Soodi and S. P. Kumar (2012), Design of low-cost hardware for lung sound acquisition and determination of inspiratory-expiratory phase using respiratory waveform, Third International Conference on Computing, Communication and Networking Technologies, pp. 1-5.

[18] X. Y. Liu, M. O'Brien, M. Mwangi, X. J. Li and G. M. Whitesides (2011), Paper-based piezoresistive MEMS force sensors. IEEE 24th International Conference on Micro Electro Mechanical Systems, Cancun, pp. 133-136.

[19] A. J. Shaw, B. A. Davis, M. J. Collins and L. G. Carney (2009), A technique to measure eyelid pressure using piezoresistive sensors. in IEEE Trans. Biomedical Engineering, vol. 56, no. 10, pp. 2512-2517.

[20] Tuantranont, T. Lomas, K. Jaruwongrungsee, A. Jomphoak and A. Wisitsoraat (2008), "Symmetrical polymumps-based piezoresistive microcantilever sensors with on-chip temperature compensation formicrofluidics applications . in IEEE Sensors Journal, vol. 8, no. 5, pp. 543-547.

[21] J. C. Suhling and R. C. Jaeger (2001), Silicon piezoresistive stress sensors and their application in electronic packaging . in IEEE Sensors Journal, vol. 1, no. 1, pp. 14-30.

[22] Wenkui Dong a, Wengui Li a, Luming Shen b, Daichao Sheng a (2019), Piezoresistive behaviors of carbon black cement-based sensors with layer-distributed conductive rubber fibers, Materials and Design. 